Manuscript received June 10, 2016; accepted for publication November 22, 2016; published online November 27, 2017.

1 Dept. of Civil and Architectural Engineering, Royal Inst. of Technology, Stockholm, Sweden

2 Dept. of Solid Mechanics, Royal Inst. of Technology, Stockholm, Sweden; and Atlas Copco, Örebro, Sweden (Corresponding author), e-mail: msaadati@kth.se

${ }^{3}$ Laboratory for Macromolecules and Bioimaging (LSB), Paul Scherrer Institut, Villigen-PSI, Switzerland; and Inst. for Biomedical Engineering, ETHZ, Zurich, Switzerland

${ }^{4}$ Dept. of Solid Mechanics, Royal Inst. of Technology, Stockholm, Sweden
D. Jelagin, ${ }^{1}$ M. Saadati, ${ }^{2}$ I. Jerjen, ${ }^{3}$ and P.-L. Larsson ${ }^{4}$

\section{Mechanical Characterization of Granite Rock Materials: On the Influence from Pre-Existing Defects}

\section{Reference}

Jelagin, D., Saadati, M., Jerjen, I., and Larsson, P.-L., "Mechanical Characterization of Granite Rock Materials: On the Influence from Pre-Existing Defects," Journal of Testing and Evaluation, Vol. 46, No. 2, 2018, pp. 540-548, https://doi.org/10.1520/JTE20160304. ISSN 0090-3973

\section{ABSTRACT}

The length, orientation, and population of pre-existing cracks play an important role in the mechanical response of quasi-brittle materials, such as granite. Specifically, Bohus granite rock is at issue in the present investigation. The aims of this study are (1) to demonstrate the existence and characteristics of pre-existing defects (cracks) in granite rock specimens, and (2) to determine the influence from these defects at characterization of such materials. In doing so, $X$ ray tomography was the method used to visualize the cracking of three-point bending specimens in the context of pre-existing defects. It was also the intention to relate the experimentally determined effect of these cracks at three-point bending testing with corresponding results from numerical methods and specifically finite-element calculations. In the latter case, the location of these cracks was of primary interest and it was shown that this feature can be of considerable importance at material characterization.

\section{Keywords}

mechanical characterization, X-ray computed tomography, pre-existing defects, granite, three-point bending, finite-element simulations

\section{Introduction}

An accurate description of the constitutive behavior of granite is of crucial importance in a number of engineering applications (e.g., drilling and cutting). Evaluation of granite materials for engineering purpose is not an easy task because granite is a very inhomogeneous material also containing many different types of defects. A particular issue in this context is the fact that during manufacturing of rock products (e.g., rock tiles, specimens for testing purposes) cracks are very easily introduced in the material. Such cracks are in this study referred to as pre-existing cracks or defects, meaning they were present before testing.

Even without these pre-existing cracks constitutive modeling of granite is a very involved matter. For one thing, it is well known that semi-brittle materials, such as rocks, behave differently in compression 
and tension [1]. Therefore, the constitutive specification for such materials should account for this difference and should be able to distinguish between the two stress-sign-dependent responses. For this purpose, Forquin and Hild [2] studied dynamic fragmentation in brittle materials because of impact loadings by using a probabilistic approach to describe the material behavior. The constitutive equation consists of a plasticity model introduced by Krieg [3] and Swenson and Taylor [4] (referred to as KST) for compressive loadings, coupled with the anisotropic damage model developed by Denoual and Hild [5], and Forquin and Hild [2] (referred to as DFH), in tension. This type of model was able to describe the fragmentation of two limestones subjected to edge-on impact (EOI) testing (Edge-on Impact) [6] and the enhancement of strength and failure pattern of a micro-concrete [7,8] and a standard concrete [9] in spalling and EOI experiments. Saadati et al. [10] applied the KST-DFH model on granite and investigated the rock fragmentation process and the force-penetration response at percussive drilling. The KST-DFH model parameters for Bohus granite have been determined based on previous experiments [11]. It should be mentioned in this context though that the number of material parameters in the KST-DFH model is quite large and extensive experimental efforts are needed to fully characterize materials based on this model.

Because of the considerable difficulties and efforts involved during characterization of granite (and other rock materials), it is important to ensure the experimental results are not influenced by features not directly related to the constitutive behavior. One of these features is the previously mentioned pre-existing cracks. Such defects are particularly of interest for granite as they are rather easily introduced at for example manufacturing. As reported by Saadati et al. [11], based on measurements performed in the tensile mode and at three-point bending tests, Bohus granite specimens exhibit two distinctly different types of behavior that somewhat loosely can be described as weak and strong based on the values on stiffness and strength. It was hypothesized by Saadati et al. [11] that this difference in behavior may be attributed to the effect of pre-existing cracks in the material. The influence of pre-existing cracks on the granite behavior has furthermore been examined numerically by Saadati et al. $[10,12]$. In particular, it is shown in Ref. [12] that introducing pre-existing cracks improves agreement with experimental observations at EOI testing.

From the above discussion, it may be concluded that preexisting cracks may have a profound influence on the material response in both testing and engineering applications. A detailed quantitative investigation and characterization of such cracks and their corresponding effect on the mechanical behavior of granite has not been attempted yet. It is the aim of this paper to remedy this shortcoming. The specimens of Bohus granite were examined with X-ray computed tomography (CT) and morphological characteristics of pre-existing defects in the material (i.e., their frequency, location, geometry, and shape) were obtained. The specimens are described in detail below in the next section. Following this investigation, a finite-element (FE) model of three-point bending testing was developed employing an elastic-strain softening constitutive model and used to examine the influence of the pre-existing defects identified with $\mathrm{X}$-ray CT on the response of the specimens. The results were compared with experimental observations to assess the influence of preexisting defect size and position on the material response. It should be commented upon the fact that a simplified constitutive model was used in the FE calculations. The main reason for this is of course computational efficiency, remembering that the KST-DFH model requires considerable numerical efforts. Furthermore, the KST-DFH model is developed mainly for dynamic problems and, in the present quasi-static situation, a simplified elastic-strain softening constitutive model is sufficient.

Finally, it should be emphasized that percussive drilling, the main application of interest in Refs. [10] and [12], is a type of loading that causes high local strain rates in the rock and makes the problem transient dynamic. For one thing, it was shown in Refs. [10] and [12] that pre-existing cracks in granite have a significant effect on the mechanical response and fracture pattern at drilling. This effect was studied in more detail in Ref. [12], using the finite element method (FEM), exploring the influence of preexisting cracks on penetration stiffness and fracture pattern in the rock at percussive drilling. These cracks may result from preimpact of the drill bit prior to drilling, or by means of other methods such as microwave and laser that are used to facilitate the drilling process. The drilling efficiency was compared for preexisting cracks with different lengths and orientations. All of the results reported in Ref. [12] concerned the Bohus granite rock characterized in Ref. [11]. It was shown that the effect of preexisting cracks is substantial and always facilitating the drilling process regardless of the crack orientation.

\section{Experimental Characterization of the Granite Specimens}

In the study by Saadati et al. [11], granite was characterized mechanically by uniaxial tensile and other testing to fully describe the material based on the KST-DFH model. It was then possible to distinguish two different stress-strain curves as shown in Fig. 1, pertinent to what could be described as strong and weak behavior. Typically, the strong specimens are approximately twice as stiff and have twice as high tensile strength when compared to the weak ones. The results in Ref. [11] are attributed to the effect from pre-existing cracks in the material, i.e., not an effect related to the constitutive behavior of granite. It should be emphasized that the present study was made to clarify the effect from pre-existing cracks at material characterization (and in other situations), not to perform a parametric study for a complete quantitative 
FIG. 1 Axial stress-strain behavior of granite at uniaxial tensile testing. The results are taken from Ref. [11].

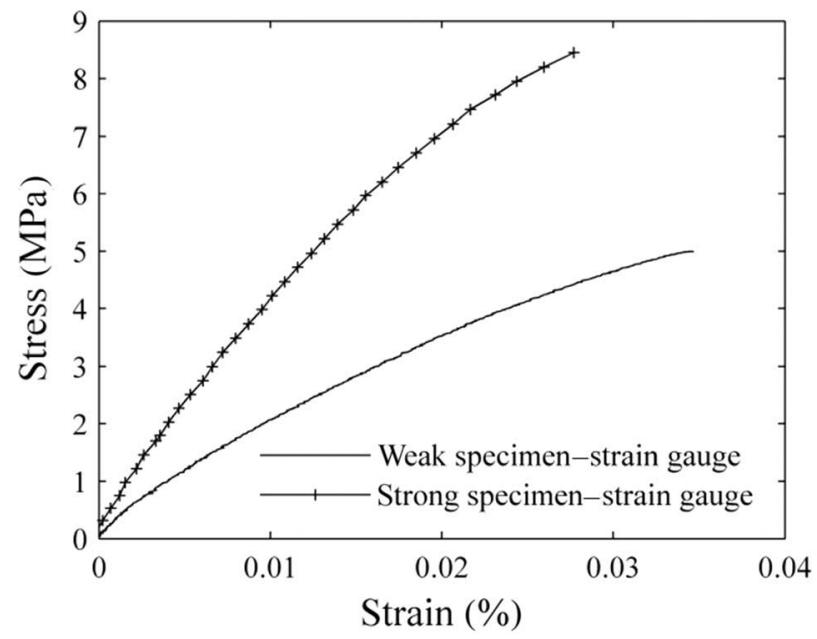

description of the influence from pre-damage in granite. Therefore, the number of experiments performed presently were limited but were supplemented with finite-element results to be described below. Indeed, finite-element analysis was a more reliable tool for determining the influence from factors such as size and position of these cracks. It should also be mentioned that when it comes to such questions (e.g., specimen preparations), this is described in detail in Saadati et al. [11].

Uniaxial tensile testing can in the present context be described as a global test in the meaning that the presence of sufficiently large pre-existing cracks will always influence the stressstrain curve regardless of the positions of these defects. On the other hand, a three-point bending test is a considerably more local test with tensile failure being initiated at the specimen surface in the middle of the specimen. However, to constitutively characterize granite (and other rock materials), such tests are necessary to perform. To evaluate the quasi-static strength of Bohus granite (and its distribution), three-point bending tests were carried out previously [11] where the nominal stress was defined based on Bernoulli beam theory from the actuator force $N$ and the support distance $l$ by:

$$
\sigma_{N}=\frac{6 N l}{4 b h^{2}}
$$

where:

$h=$ the height of the specimen, and

$b=$ the width of the specimen.

The nominal stress-strain curve in most of the specimens in the above study was mainly linear before maximum load, followed by a brittle behavior leading to final failure. Based on strain gauge measurements, the failure strain pertinent to these specimens was approximately 0.0006 . However, some specimens showed significant non-linearity in the nominal stress-strain curve with a noticeably lower load capacity (and also a failure strain that depends on the length of the strain gauges) compared with the others and this type of response was related to the effect from pre-existing cracks in the specimens [11]. This hypothesized effect from pre-existing cracks remains, even though very plausible results based on previous results in Refs. [10] and [12] are not proven. It was the aim of the present investigation to validate it by confirming its existence by X-ray tomography and explaining in detail its influence on the mechanical behavior at threepoint bending testing.

To perform a detailed characterization of such cracks, the broken specimens from three-point bending tests performed in an earlier study [11] were examined using X-ray tomography. All of the specimens loaded in three-point bending [11] had a square cross section of $20 \times 20 \mathrm{~mm}$ and length of $100 \mathrm{~mm}$. These specimens belong to both groups of lower load capacity, S16730 and S16727 (with nominal tensile strength levels 9.47 and 9.49 $\mathrm{MPa}$ respectively) and higher load capacity, S16735 and S16740 (with nominal tensile strength levels 19.18 and $20.08 \mathrm{MPa}$, respectively). It should be noted that around 30 three-point bending tests were performed and the scatter in the results were captured and reported previously in Ref. [11]. Presently, in this study, a few weak and strong specimens from that investigation were selected for tomography.

In the X-ray computed tomography investigation, the specimens were broken in a three-point bending test and then onehalf of each specimen was investigated by a custom-made micro computed tomography (CT) setup at Empa (Swiss Federal Laboratories for Materials Science and Technology). The scanned specimens had a square cross section of $20 \times 20 \mathrm{~mm}$ and a length of 50 to $65 \mathrm{~mm}$ (Fig. 2). The CT setup consists of an X-ray tube from Viscom (XT9160-TXD), a flat-panel X-ray detector from Perkin Elmer (XRD 1621 CN3 ES, $200 \mu \mathrm{m}$ pixel size), and a high precision rotation stage (UPR-160 F air) mounted on three linear

FIG. 2 Typical Bohus granite specimen measured with X-ray CT.

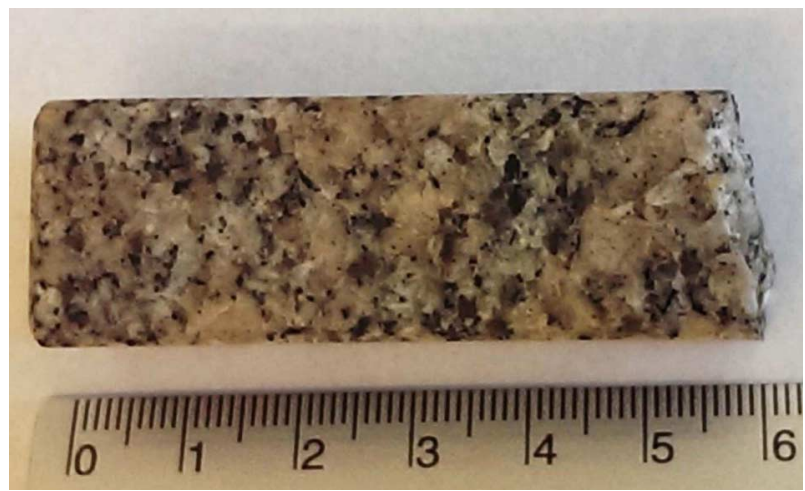


stages (LS-270) from Micos. The X-ray tube current was $80 \mu \mathrm{A}$ and the tension was $140 \mathrm{kV}$. Two samples were mounted together inside a poly(methyl methacrylate) (PMMA) cylinder, one on top of the other, and then fixed on the rotation table between the Xray source and detector. The distance between the source and detector was $1017.3 \mathrm{~mm}$ and the samples were mounted at $76.3 \mathrm{~mm}$ from the source. By this cone beam geometry, a magnification of 13.3 was achieved. For each sample, two scans were executed to obtain a good spatial resolution. For each scan, 1440 projection images with $2000 \times 2040$ pixels distributed over $360^{\circ}$ were recorded with an individual exposure time of $4.8 \mathrm{~s}$. Because the response of the detector was linearized beforehand, the projection data were corrected only for ring- and beam-hardening artefacts before the three-dimensional distribution of the attenuation coefficient was calculated by a self-developed Feldkamp algorithm [13]. The obtained voxel size was $15 \mu \mathrm{m}$.

\section{Computational Study}

The X-ray tomography study described above concerns identification and characterization of pre-existing cracks in granite. To determine its influence on the mechanical behavior because of size and location, and in particular the three-point bending test as discussed above, finite-element methods using the commercial FE-package ABAQUS [14] was used.

As discussed above, for computational reasons (and the fact that dynamic effects were not considered presently), it was considered unnecessary to adhere to the KST-DFH model for constitutive specification. Instead, the results from the uniaxial tensile experiments reported in [11] were used to calibrate a simple strain-dependent damage evolution law [15] that is applicable for materials such as rock and concrete developing damage in the form of microcracks. Explicitly, this damage law reads:

$$
\omega=\left\{\begin{array}{cc}
\left(\frac{\varepsilon_{\max }-\varepsilon_{0}}{\varepsilon_{c}-\varepsilon_{0}}\right)^{s} & \varepsilon_{\max } \geq \varepsilon_{0} \\
0 & \varepsilon_{\max }<\varepsilon_{0}
\end{array}\right.
$$

where:

$\varepsilon_{\max }=$ the maximum principal strain,

$\varepsilon_{c}=$ the fracture strain,

$s=$ a material-dependent constant, and

$\varepsilon_{0}=$ the strain value that was damage initiated.

Using the constitutive law for linear elastic materials with stress $\sigma$, strain $\varepsilon$, and isotropic damage parameter $\omega$ in uniaxial form:

$$
\sigma=(1-\omega) E \varepsilon
$$

where:

the parameters $\varepsilon_{c}=0.0019, s=0.2, \varepsilon_{0}=0$, and $E=50 \mathrm{GPa}$ are obtained from curve fitting of uniaxial tensile test results reported in Ref. [11].
FIG. 3 Location and size of the cracks analyzed in the finite-element calculations.

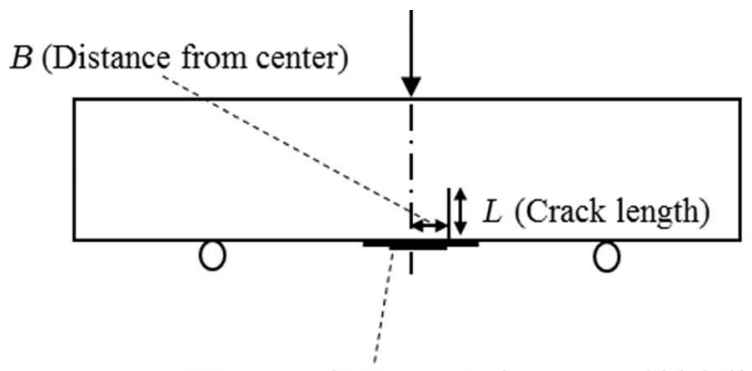

$10 \mathrm{~mm}$ and $20 \mathrm{~mm}$ strain gauges (thick lines)

One obtains a similar stress-strain behavior as the uniaxial tensile experiment in Ref. [11]. First, this constitutive model was used to simulate the three-point bending test and the average strain at the strain gauges of length $10 \mathrm{~mm}$ and $20 \mathrm{~mm}$ (mounted on the center of the tensile surface of the specimen) were compared to the experiment. However, in this case, the simulation results did not match the experimental ones. In another effort, physical cracks with different lengths and distances from the center of the specimens were introduced, and a parameter study pertinent to the length and location of the crack was performed to obtain as close values of strain as possible to the experiment for both $10 \mathrm{~mm}$ and $20 \mathrm{~mm}$ gauges (Fig. 3). In case of off-center cracks, two identical cracks were introduced on opposite sides of, with equal distance from, the symmetry line of the beam. This was done for simplicity to retain symmetry. It should also be mentioned that the simulations were performed in a forcecontrol manner. Therefore, the force was increased progressively in an analysis until a certain force level, corresponding to the maximum force from the experiment, was achieved. At that instance, the simulations were stopped and the displacements (strains) were recorded.

The half-symmetry discretized geometry of the three-point bending specimen is shown in Fig. 4 with eight-noded planestrain elements. In the cases when a physical crack is included in the model, the symmetry boundary condition along the crack length was removed (Fig. 4). It is assumed that the depth of the cracks in the simulations is the same as the depth of the specimens. This is an obvious fact remembering that plane-strain conditions are adhered to.

\section{Results and Discussion}

The results from the X-ray tomography investigation for a part of a typical vertical slice through the specimen is shown in Fig. 5a. In general, very few cracks branching from the main failure plane were observed. As seen in Fig. 5 there are two smaller cracks (highlighted with arrows) located at approximately $12-\mathrm{mm}$ 
FIG. 4

Typical finite-element mesh used in the numerical calculations of the specimen with center crack. Symmetry conditions are indicated.

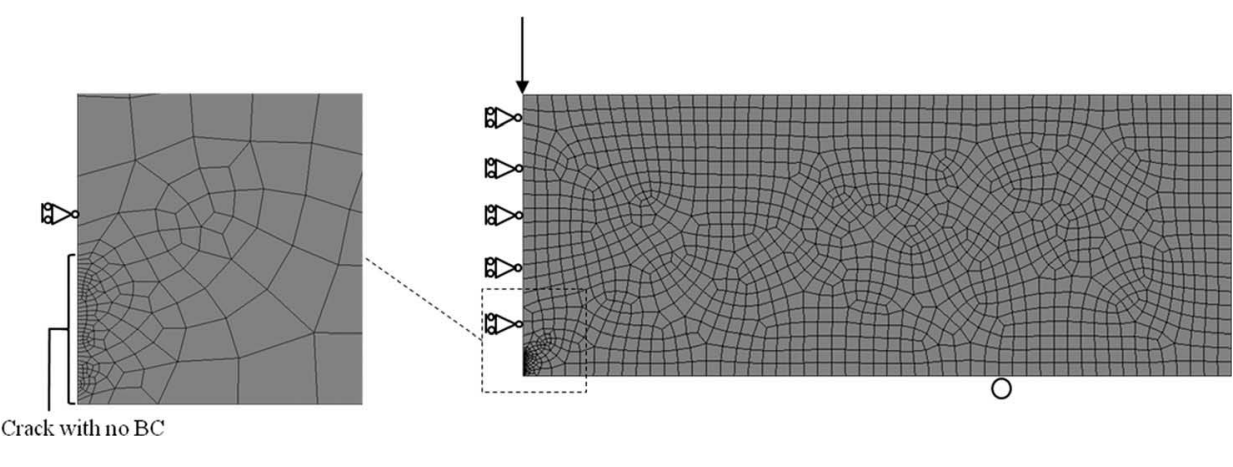

distance from the breakage plane. A close-up view of those secondary cracks, i.e., the region inside the square frame in Fig. $\mathbf{5 a}$, is given in Fig. 5b. As the secondary cracks observed in Fig. $\mathbf{5 b}$ initiate away from the region of high tensile stress induced in threepoint bending test, it is likely that either those cracks were preexisting before the test or propagated during the test from smaller pre-existing cracks. It might also be speculated that the two cracks in Fig. 5b are in fact segments of one crack with a closed segment in between. The cracks were, however, followed through the thickness of the specimen and no visual proof of their

FIG. 5 Vertical slice through specimen S16730: (a) main failure plane along with secondary cracks (highlighted with arrows) starting at the specimen surface approximately $12 \mathrm{~mm}$ from the breakage site, and (b) close-up view of the region with the secondary cracks.

(a)

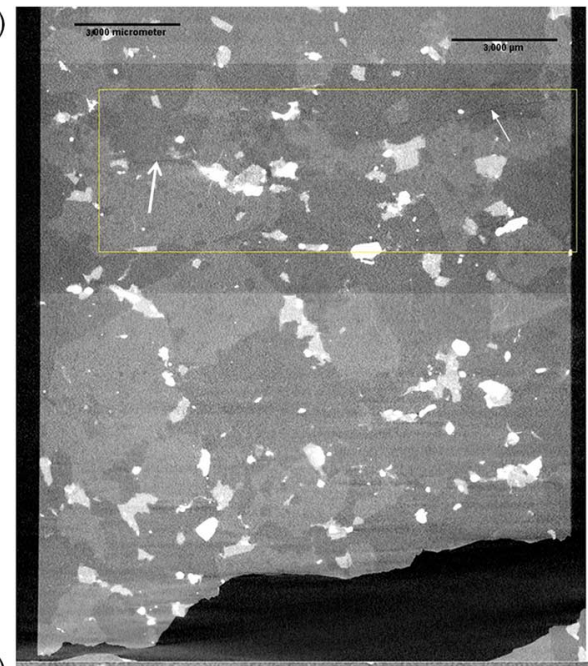

(b)

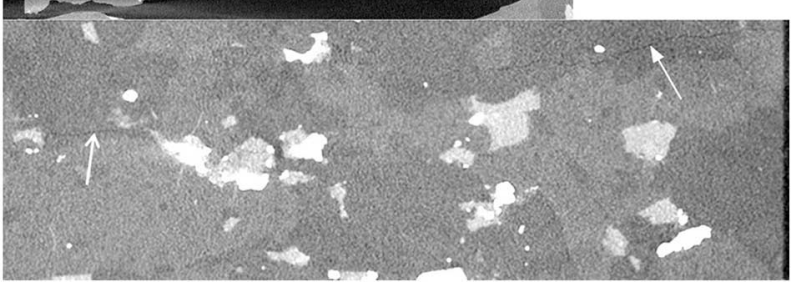

connectivity was found. The geometry of the secondary cracks in Fig. 5 has been characterized as follows. Crack evolution was examined visually, and, at the slice where the crack was longest, it was traced manually with a freehand line; the length of the trace line was considered to correspond to the maximum crack length. The crack depth (crack extension perpendicular to the length direction) was obtained by multiplying number of slices where the crack was visible with the resolution of the scan. The crack shown in the right part of Fig. 5b was measured to have a maximum length of $5 \mathrm{~mm}$ and a depth of $1.6 \mathrm{~mm}$.

To examine the distribution of the pre-existing defects in the specimens, the attention is now focused on the approximately 25 mm-long specimen segment starting from the undamaged end. As this region was not subjected to high tensile stresses during testing, the defects found there are not likely to be induced during the test. Generally, three types of defects were found in the examined tomography pictures: (1) rounded pores, (2) smaller cracks with a maximum opening below $15 \mu \mathrm{m}$ (i.e., below voxel size), and (3) larger cracks with an opening above $15 \mu \mathrm{m}$; these defects are referred to in the discussion below as Type 1, 2, and 3 correspondingly.

In Fig. $\mathbf{6 a}$ and $\mathbf{6 b}$, the horizontal cross sections of specimens S16740 and S16727 are shown; the slices shown in Fig. $\mathbf{6 a}$ and $\mathbf{6 b}$ are located at distances of 18 and $7 \mathrm{~mm}$ from the undamaged ends of the specimens, respectively. As shown in Fig. 6, the specimens contain rounded pores, i.e., Type 1 defects and sharp cracks, i.e., Type 3 defects, highlighted with circle and arrow, respectively. The cracks shown in Fig. 6 originate from the specimen edge and have most likely been induced during specimen manufacturing. The cracks shown in Fig. 6 are quite large with maximum opening larger than voxel size, i.e., corresponding to the Type 3 defect above. Type 3 cracks have been observed in all specimens except S16735, but found to be relatively few (1-3 per specimen). The largest Type 3 crack in each specimen has been measured manually with the procedure presented above and the corresponding dimensions are reported in Table 1. Specimen pores were segmented with the thresholding operation; gray level threshold (i.e., the maximum intensity of gray level corresponding 
FIG. 6

Horizontal slices through specimens (a) S16740, and (b) S16727. Rounded pores and large secondary cracks, i.e., Type 1 and Type 3 defects (highlighted with circle and arrows, respectively).

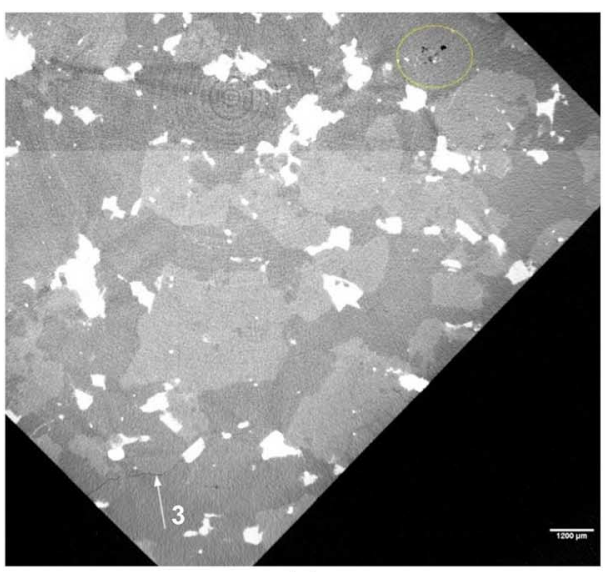

(a)

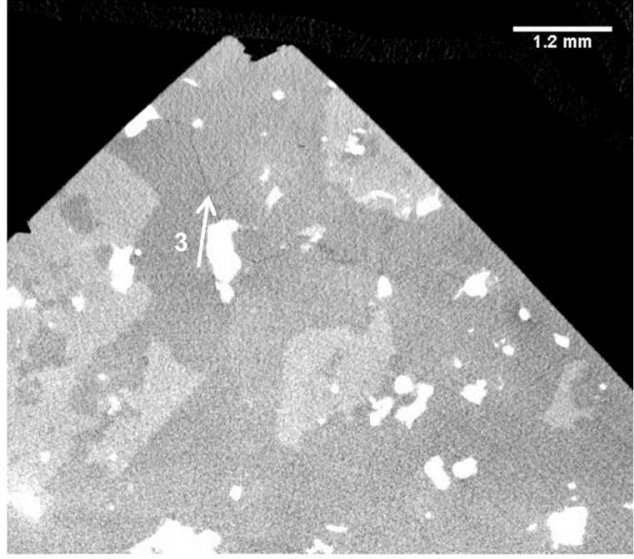

(b) to air) has been identified based on gray level outside of specimen boundaries. Quantitative analysis of specimen porosity was performed with commercial software Avizo Fire, and found to be less than $0.1 \%$ with maximum and mean porosity values presented in Table 1.

In all four specimens, a significant number of small cracks with an opening smaller than scan resolution (i.e., Type 2 defects) were found (the opening cannot be determined, but it can be detected within the size of the voxel); two typical examples are shown in Fig. 7. The Type 2 cracks are visible in CT data as linear features with the gray level in between the one corresponding to air and the one corresponding to the surrounding rock. Because of low contrast with the surrounding rock material, it was difficult to measure these cracks in a reliable manner. General observations pertinent to Type 2 cracks may, however, be summarized as follows: as seen in Fig. 7, these cracks appear to start from defects on the specimen surface, their length was found to be approximately between 0.1 and $1 \mathrm{~mm}$, and their width was less than $15 \mu \mathrm{m}$.

As it has been previously reported in Ref. [12], based on numerical simulations of edge-on impact tests and of percussive drilling operations, incorporating pre-existing cracks of $2 \mathrm{~mm}$ length will result in improved qualitative and quantitative agreement with experimental observations. X-ray CT measurements performed presently confirmed the presence of different types of defects in the material. In particular, smaller cracks initiating at specimen edges, shown in Fig. 7, were found in all specimens. Quantitative characteristics of specimen defects obtained presently and shown in Table 1 do not, however, correlate with the experimentally measured tensile strengths of specimens reported above. That may be because of the fact that the geometry of the pre-existing defects has been affected during the threepoint bending test. Furthermore, as the tensile stress state induced in the three-point bending tests is highly localized, the measured specimen tensile strength will be primarily affected by the preexisting defects in the vicinity of the main failure plane. As Type 2 cracks have been observed in all of the specimens, it may also be hypothesized that the location of these defects with respect to the zone of maximum tension induced during the test controls the measured tensile strength. This hypothesis was accordingly tested by the above-described FEM simulations of three-point bending tests of granite specimens. It would also be interesting to isolate the cracks at the specimen edges/ends and then compare the crack density between the different types of specimens. This is, however, left for future studies.

In Fig. 8, the experimentally measured response for one of the weak specimens is shown along with the computational results obtained for the specimens without pre-existing defect and with Type 2 crack of $0.85 \mathrm{~mm}$ length located in the middle of the specimen. As shown in Fig. 8, the presence of a $0.85-\mathrm{mm}$ crack results in a qualitative change of the material's response much closer to the experimental ones. Namely, as the pre-existing crack starts to propagate, the specimen response becomes highly non-linear and a discrepancy between the strains measured at the 10- and 20-mm strain gauge appears (Fig. 3). The resulting failure strain also

TABLE 1 Specimen pore volume and crack length.

\begin{tabular}{lccc}
\hline Specimen No. (Specimen Characteristic) & S16727 (Weak Specimen) & S16730 (Weak Specimen) & S16735 (Strong Specimen) \\
\hline Mean pore volume $\left(\mathrm{mm}^{3}\right)$ & $2 \times 10^{-5}$ & $2 \times 10^{-4}$ & $4 \times 10^{-5}$ \\
Max pore volume $\left(\mathrm{mm}^{3}\right)$ & $4.2 \times 10^{-3}$ & $1 \times 10^{-2}$ & $1 \times 10^{-4}$ \\
Maximum crack dimensions $L(\mathrm{~mm}), W(\mathrm{~mm})$ & $7.4,0.3$ & $3.3,0.6$ & $6 \times 10^{-3}$ \\
\hline
\end{tabular}


FIG. 7

Horizontal slices through specimens (a) S16727, and (b) S16735. Smaller secondary cracks (i.e., Type 2 defects) are highlighted with arrows.

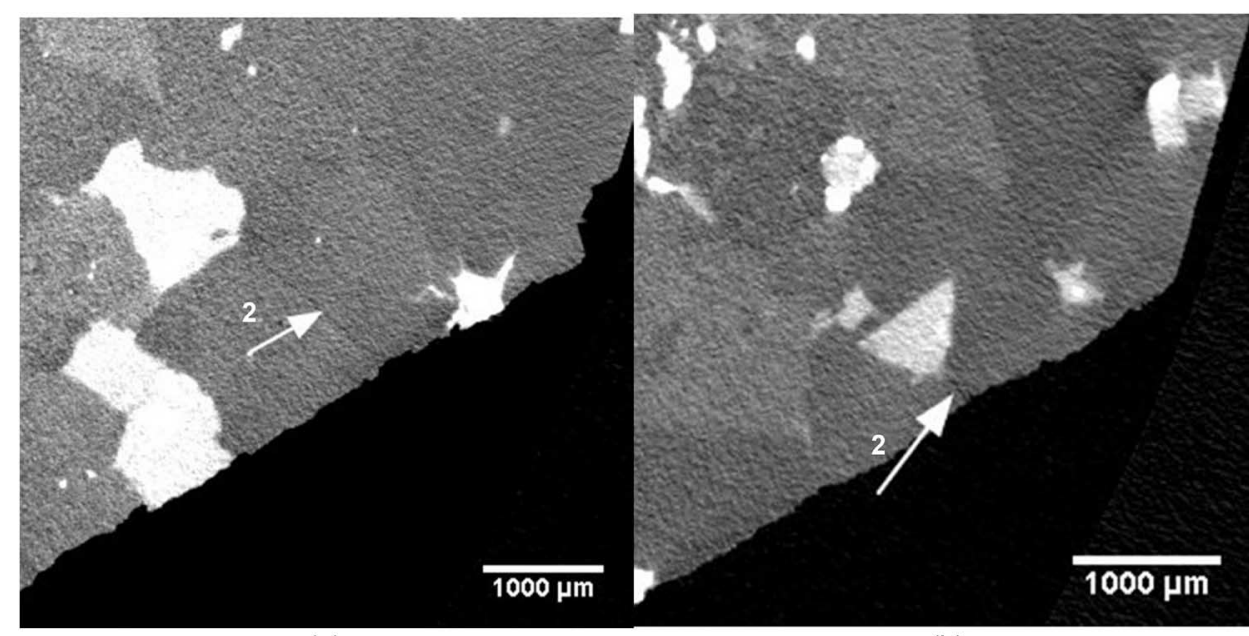

(a)

(b)

FIG. 8

Experimental and computational (FEM) results for the force-strain response pertinent to three-point bending tests. Experimental results and computational (FEM) results for specimens with no crack and with a center $\operatorname{crack}(B=0)$ with length $L=0.85 \mathrm{~mm}$. The point of failure is indicated by the end point of the curves.

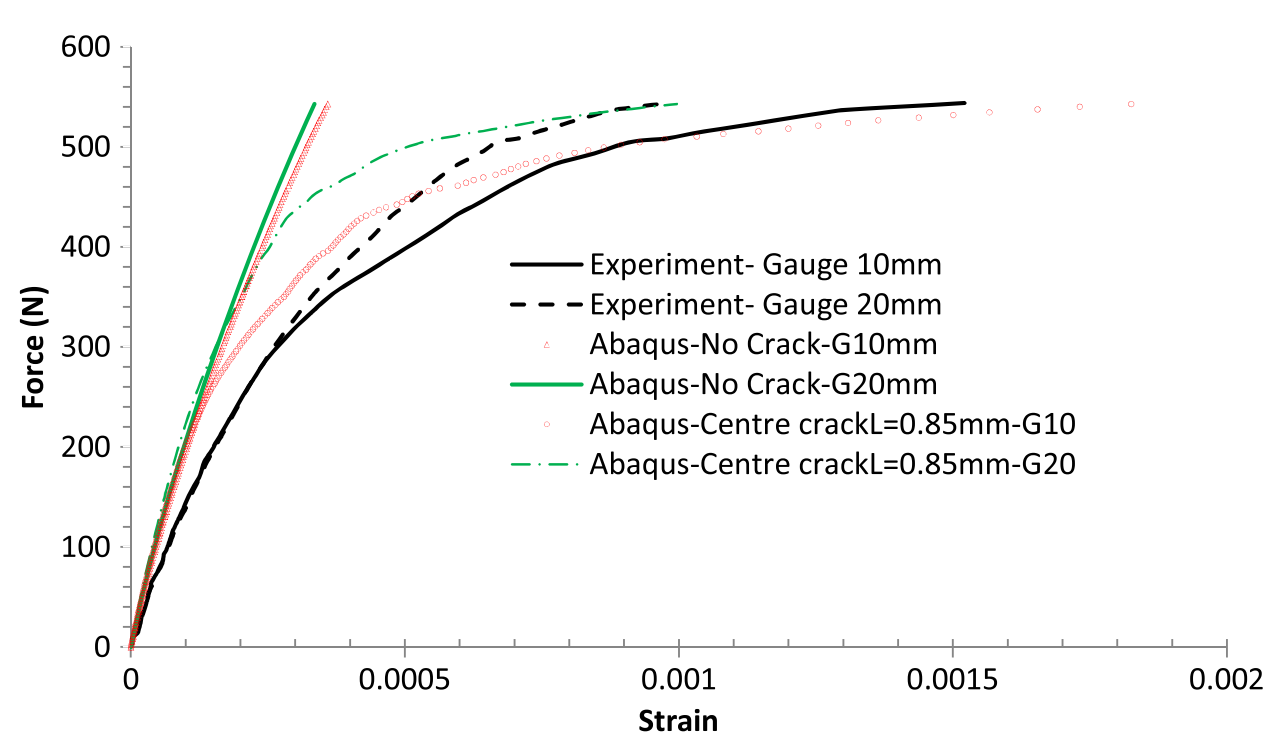

increases by approximately a factor of 5 , which is in at least qualitative agreement with the experimental observations reported above. At the same time, the initial stiffness of the specimen is not affected by the presence of the crack and can most likely be explained by the use of a simplified constitutive equation calibrated based on uniaxial tensile testing.

In Fig. 9, the influence of pre-existing crack size on the specimen tensile strain at failure is shown for the crack located in the middle of the specimen. Results in Fig. 9 are given for the initial crack lengths of $0.7,0.85$, to $1 \mathrm{~mm}$, i.e., the upper range of Type 2 defect size. As shown in Fig. 9, although the pre-existing crack always results in higher strain at failure, a certain crack length is needed to explain the experimental observations.

In Fig. 10, the results are reported for the specimens containing the pre-existing crack of $0.85 \mathrm{~mm}$ located at various distances from the specimen center line. It should be noted that, in the calculations, it is assumed that two identical off-center cracks are situated on opposite sides of, with equal distance from, the symmetry line of the beam. As shown in Fig. 10, changing the crack position affects the results profoundly; the maximum strain at failure is reduced by a factor of 2.5 by moving the crack $3.5 \mathrm{~mm}$ from the center line. As reported above, the Type 2 defects were found to be $1 \mathrm{~mm}$ maximum in length; based on the simulation results, it may be argued that presence of the Type 2 defect within a certain distance of the center line will result in experimental observations corresponding to a so-called weak specimen.

Accordingly, it can be stated that both the size and location of pre-existing defects are of importance at material characterization by three-point bending testing (and, of course, 
FIG. 9

Experimental and computational (FEM) results for the force-strain response pertinent to three-point bending tests. Experimental results and computational (FEM) results for specimens with center cracks $(B=0)$ with, length $L=0.7$,

0.85 , and $1 \mathrm{~mm}$. The point of failure is indicated by the end point of the curves.

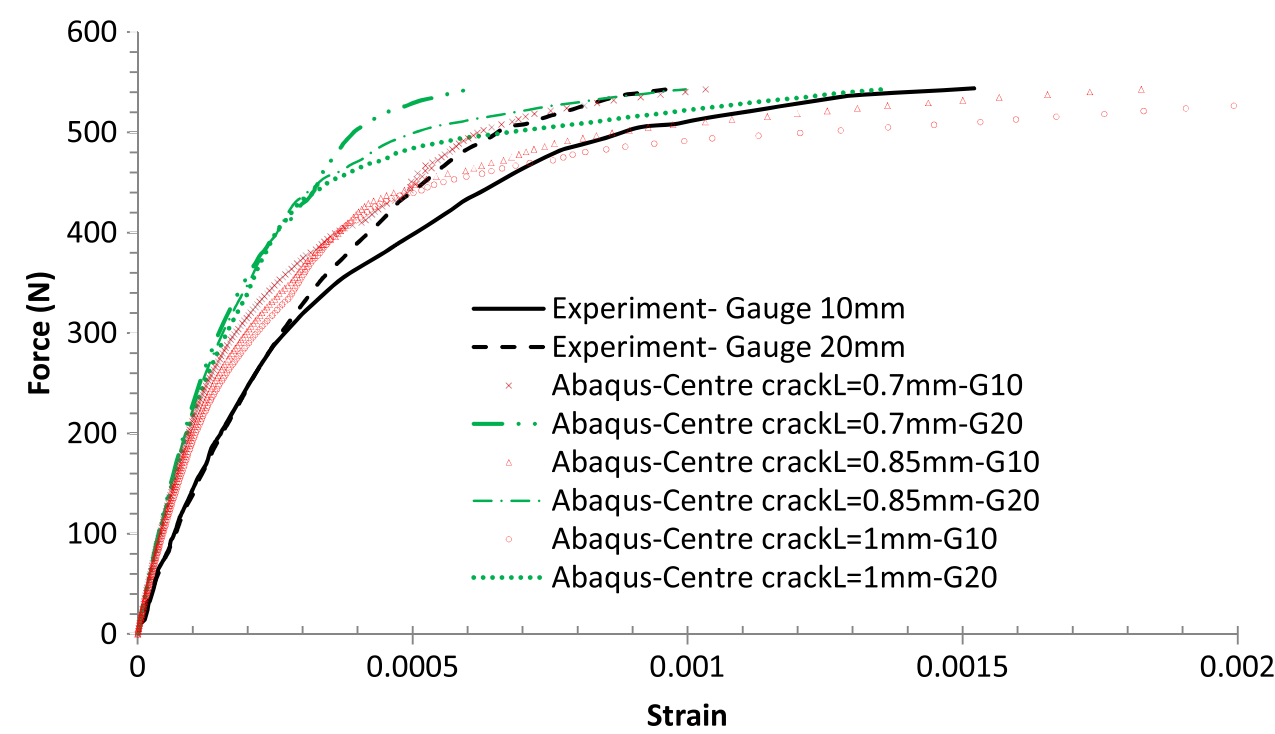

FIG. 10

Experimental and computational (FEM) results for the force-strain response pertinent to three-point bending tests. Experimental results and computational (FEM) results for specimens with offcenter cracks ( $B=3.5,4.5$, and $5.5 \mathrm{~mm}$ ) with length $L=0.85 \mathrm{~mm}$. The point of failure is indicated by the end point of the curves.

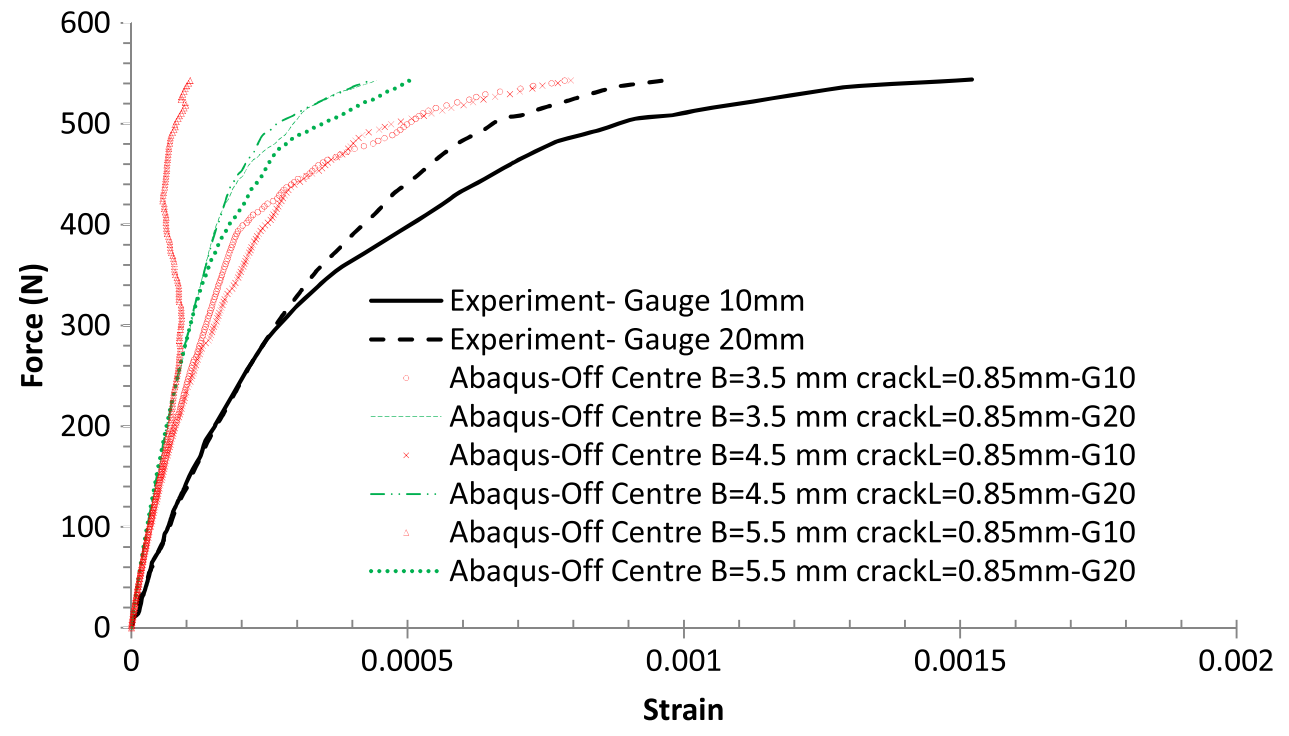

also in uniaxial tensile testing). Thus, it is of profound importance in such a situation to perform a sufficient amount of experiments (on many specimens) to ensure results pertinent to non-cracked specimens and, thereby, in a reliable manner determine the true material properties of the homogeneous granite material.

\section{Concluding Remarks}

Defects are easily introduced into granite material during manufacturing. In the present study, such defects are investigated with mainly its influence at material characterization in mind. The most important outcomes of this study are:

- The existence of pre-existing defects (defects present before testing) are established from X-ray tomography observations.

- It is shown from three-point bending tests that such defects can severely influence the experimental observations pertinent to material characterization of granite materials.

- The experimental results are verified and quantified by FEM calculations.

- The main remedy to avoid the influence from these defects is to perform a large number of experiments on different 
specimens to ensure results pertinent to non-cracked specimens.

\section{References}

[1] Jaeger, J. C., Cook, N. G. W., and Zimmerman, R., Fundamentals of Rock Mechanics, Wiley, New York, 2009.

[2] Forquin, P. and Hild, F., "A Probabilistic Damage Model of the Dynamic Fragmentation Process in Brittle Materials," Adv. Appl. Mech., Vol. 44, 2010, pp. 1-72, https://doi.org/ 10.1016/S0065-2156(10)44001-6

[3] Krieg, R. D., "A Simple Constitutive Description for Soils and Crushable Foams," Report SC-DR-7260883, Sandia National Laboratory, Albuquerque, NM, 1978.

[4] Swenson, D. V. and Taylor, L. M., "A Finite Element Model for the Analysis of Tailored Pulse Stimulation of Boreholes," Int. J. Numer. Anal. Methods Geomech., Vol. 7, No. 4, 1983, pp. 469-484, https://doi.org/10.1002/nag.1610070408

[5] Denoual, C. and Hild, F., "A Damage Model for the Dynamic Fragmentation of Brittle Solids," Comput. Methods Appl. Mech. Eng., Vol. 183, No. 3, 2000, pp. 247-258, https:// doi.org/10.1016/S0045-7825(99)00221-2

[6] Grange, S., Forquin, P., Mencacci, S., and Hild, F., "On the Dynamic Fragmentation of Two Limestones Using Edge-On Impact Tests," Int. J. Impact Eng., Vol. 35, No. 9, 2008, pp. 977-991, https://doi.org/10.1016/ j.ijimpeng.2007.07.006

[7] Forquin, P. and Erzar, B., "Dynamic Fragmentation Process in Concrete Under Impact and Spalling Tests," Int. J. Fract., Vol. 163, Nos. 1-2, 2010, pp. 193-215.
[8] Erzar, B. and Forquin, P., “An Experimental Method to Determine the Tensile Strength of Concrete at High Rates of Strain," Exp. Mech., Vol. 50, No. 7, 2010, pp. 941-955, https://doi.org/10.1007/s11340-009-9284-Z

[9] Erzar, B. and Forquin, P., "Experiments and Mesoscopic Modelling of Dynamic Testing of Concrete," Mech. Mater., Vol. 43, No. 9, 2011, pp. 505-527, https://doi.org/ 10.1016/j.mechmat.2011.05.002

[10] Saadati, M., Forquin, P., Weddfelt, K., Larsson, P. L., and Hild, F., "Granite Rock Fragmentation at Percussive Drilling-Experimental and Numerical Investigation," Int. J. Numer. Anal. Methods Geomech., Vol. 38, No. 8, 2014, pp. 828-843, https://doi.org/10.1002/nag.2235

[11] Saadati, M., Forquin, P., Weddfelt, K., Larsson, P. L., and Hild, F., "On the Mechanical Behavior of Granite Material with Particular Emphasis on the Influence from Pre-Existing Cracks and Defects," J. Test. Eval., Vol. 46, No. 1, 2018, https://doi.org/10.1520/JTE20160072

[12] Saadati, M., Forquin, P., Weddfelt, K., Larsson, P. L., and Hild, F., "A Numerical Study of the Influence from PreExisting Cracks on Granite Rock Fragmentation at Percussive Drilling," Int. J. Numer. Anal. Methods Geomech., Vol. 39, No. 5, 2015, pp. 558-570, https://doi. org/10.1002/nag.2331

[13] Feldkamp, L. A., Davis, L. C., and Kress, J. W., "Practical Cone-Beam Algorithm," J. Opt. Soc. Am. A, Vol. 1, No. 6, 1984, pp. 612-619, https://doi.org/10.1364/JOSAA.1.000612

[14] Abaqus 6.11, "Dassault Systèmes Simulia," Providence, RI, 2011.

[15] Gudmundson, P., Material Mechanics, The Royal Institute of Technology, Stockholm, Sweden, 2006. 